\title{
Diverse target gene modifications in Plasmodium falciparum using Bxb1 integrase and an intronic attB
}

\author{
Praveen Balabaskaran-Nina ${ }^{1,2}$ and Sanjay A. Desai ${ }^{1 *}$
}

\begin{abstract}
Genetic manipulation of the human malaria parasite Plasmodium falciparum is needed to explore pathogen biology and evaluate antimalarial targets. It is, however, aggravated by a low transfection efficiency, a paucity of selectable markers and a biased A/T-rich genome. While various enabling technologies have been introduced over the past two decades, facile and broad-range modification of essential genes remains challenging. We recently devised a new application of the Bxb1 integrase strategy to meet this need through an intronic attB sequence within the gene of interest. Although this attB is silent and without effect on intron splicing or protein translation and function, it allows efficient gene modification with minimal risk of unwanted changes at other genomic sites. We describe the range of applications for this new method as well as specific cases where it is preferred over CRISPRCas9 and other technologies. The advantages and limitations of various strategies for endogenous gene editing are also discussed.
\end{abstract}

Keywords: Malaria, DNA transfection, Intron, Bxb1 integrase, Gene editing

\section{Background}

Despite advances from combination therapies and public health measures such as bednets, more than 400,000 people still die of malaria annually. Since Plasmodium falciparum, a virulent human malaria parasite, acquires resistance to most antimalarial drugs quickly, new drug targets and a better understanding of resistance mechanisms are needed to sustain advances in global health. These goals depend on transfection studies in cultured $P$. falciparum parasites [1-3]. Because transfection efficiencies are low, selectable markers are required; the available markers have facilitated functional analyses [4], but their relatively small number adds to the difficulties faced in molecular and biochemical studies of this pathogen.

Gene manipulation in this system requires single or double crossover homologous recombination into the haploid genome as the parasite lacks the enzymes to

\footnotetext{
* Correspondence: sdesai@niaid.nih.gov

${ }^{1}$ The Laboratory of Malaria and Vector Research, National Institute of Allergy and Infectious Diseases, National Institutes of Health, Rockville, MD 20852, USA

Full list of author information is available at the end of the article
}

carryout non-homologous end joining. Historically, these experiments relied on infrequent, coincidental genome breaks at or near the target site, often necessitating many months of continuous cultivation and drug-cycling to obtain detectable levels of integration [5]. Further aggravating this process, the circular plasmids required for stable retention in transfected parasites tend to concatemerize under drug selection, reducing the likelihood of integration and complicating interpretation of some phenotypes $[6,7]$.

Subsequent implementation of piggyBac transposase and mycobacteriophage Bxb1 integrase facilitated stable integration of transgenes and reporters $[8,9]$, but these approaches have not been used to edit specific genes of interest. The advent of sequence-specific nucleases then opened the door to directed gene editing in P. falciparum, with zinc finger nucleases (ZFN) and clustered regulatory interspaced short palindromic repeats (CRISPRs) successfully used to study parasite biology [10-15]. Gene editing with ZFN is expensive and requires a new enzyme design for each target locus. As with many other organisms, CRISPR-Cas9 is quickly becoming the preferred strategy because of simplicity and cost, but several important

(c) The Author(s). 2018 Open Access This article is distributed under the terms of the Creative Commons Attribution 4.0 International License (http://creativecommons.org/licenses/by/4.0/), which permits unrestricted use, distribution, and 
concerns prevent the full spectrum of molecular modifications. Among these, off-target effects due to Cas9-mediated cleavage at unwanted genomic sites and the dependence on specific recognition motifs (known as protospacer adjacent motifs or PAMs) are considered the most problematic in many organisms [16].

To address these concerns and to add another option for DNA transfection in P. falciparum, we recently implemented a novel application of the Bxb1 integrase transposition technology to permit targeted gene replacements [17]. Our approach achieves rapid site-specific integration, allows the full spectrum of native gene modifications, and has a distinct set of advantages when compared to other methods including CRISPR-Cas9. Our strategy is based on the introduction of a silent $a t t B$ element into an intron of the target gene; we found that this $40 \mathrm{bp}$ insertion is well-tolerated as it does not adversely affect either intron splicing or subsequent translation and function of the encoded protein. Once integrated, this attB element permits rapid recombination with an attP site on subsequent transfection plasmids to replace the downstream sequence with desired modifications. Although our strategy requires an additional transfection to first introduce the $a t t B$ site, it then enables an unlimited array of distal modifications including site-directed mutations, insertions, deletions, epitope tagging, and conditional knockdown through the introduction of ddFKBP or EcDHFRbased destabilization domains, the TetR aptamer module, or the glmS riboswitch [18-21]. After describing our procedures, we summarize the advantages and limitations of this approach when compared to currently available methods.

\section{Mechanism of attB-attP recombination and conventional application in $P$. falciparum}

Bxb1 integrase is a mycobacteriophage serine integrase that catalyses recombination between an attP sequence in the phage and an attB sequence in the Mycobacterium smegmatis groEL1 gene with minimal fully effective sequences of 48 and $38 \mathrm{bp}$, respectively. This recombination enables phage integration into the bacterial genome [22-24]. Recombination begins when Bxb1 dimers bind to each of these sites with high affinity $\left(K_{d}\right.$ estimated at 70 $\mathrm{nM})$; these dimers then interact with each other to form a synaptic tetramer, a process known as synapsis [25]. All four DNA strands are then cleaved within a central $8 \mathrm{bp}$ core conserved between attB and attP (Fig. 1a); cleavage occurs asymmetrically at a non-palindromic 5'-GT dinucleotide to ensure faithful exchange of phage attP and bacterial $a t t B$ sequences and prevent incorrect joining of half-sites [24]. A conserved serine in Bxb1 forms a

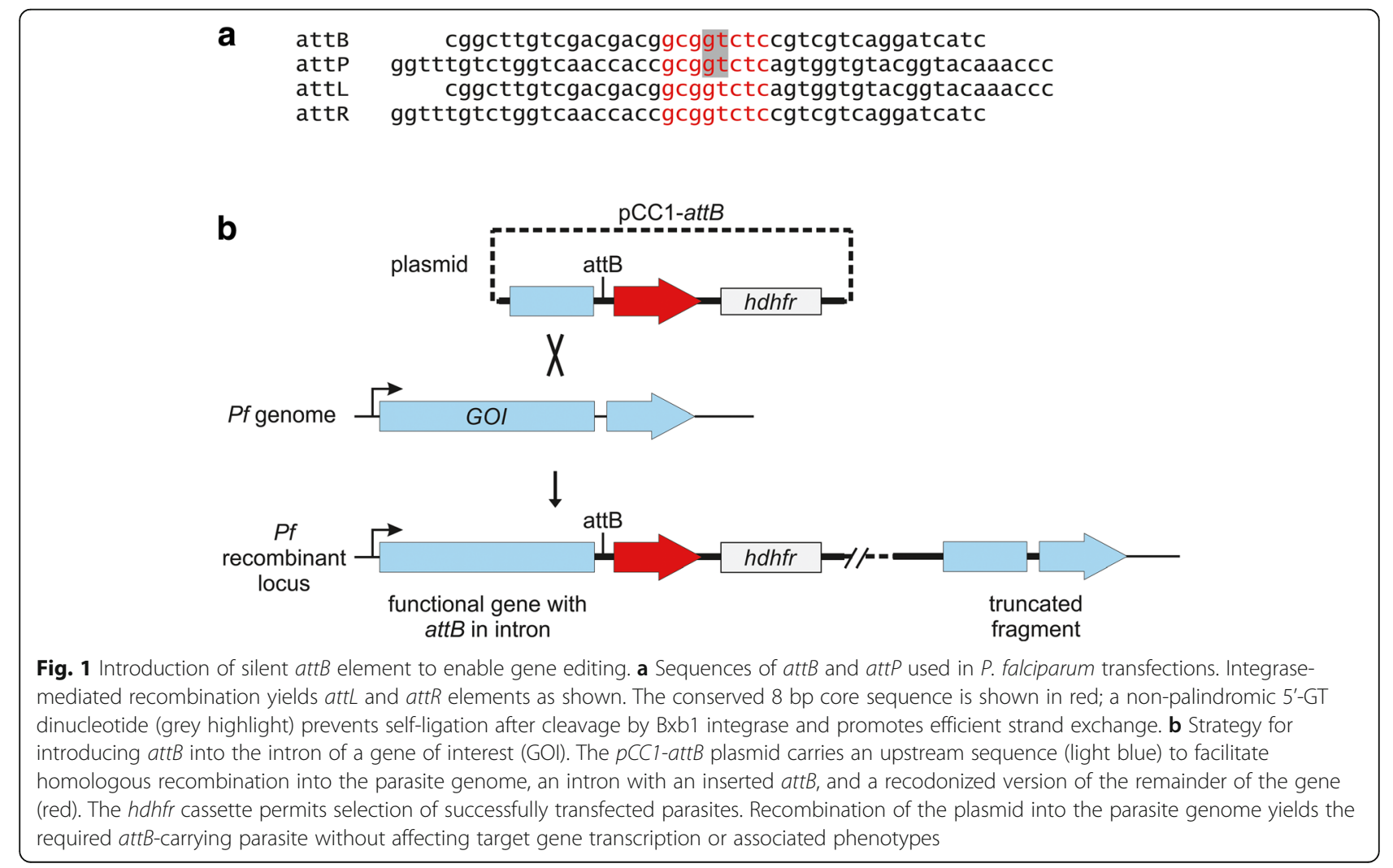


covalent bond with the cleaved DNA, allowing rotation and re-ligation of the two double-stranded DNA polymers. The resulting sites, termed attL and attR, differ from one another and do not match the original $a t t B$ and attP elements as they carry swapped flanking sequences. This reaction is generally considered irreversible, but auxiliary proteins known as the recombination directionality factors (RDFs) can facilitate the reverse reaction and excision of the phage from the host genome [26]. Structural studies have revealed extensive contacts between the recombinase domain, an unusual zinc ribbon domain, and att sequences that confer specificity for attB-attP recombination and prevent the reverse attL-attR recombination reaction [27]. Kinetic and mutagenesis experiments have led to a model of highly coordinated formation of a synaptic complex between a tetrameric recombinase complex and two att sites, controlled cleavage and rotation, hybridization and religation [28-31]. When compared to tyrosine integrases, serine integrases do not require DNA super coiling, divalent cations or bacterial host factors for integrase activity, making their use in heterologous systems more attractive.

Nkrumah et al. [9] demonstrated this system's utility in malaria parasites by showing efficient recombination of an attP-containing plasmid with an engineered $a t t B$ in the parasite genome; Bxb1 integrase was expressed from a co-transfected helper plasmid. The attB sequence, otherwise not present in P. falciparum, was introduced into the nonessential cg6 gene; several such cg6-attB lines have been generated in distinct genetic backgrounds [32]. These studies reported a rapid appearance of recombinants with a near-homogeneous population of integrant parasites within 2-4 weeks of the second transfection [9]. Nevertheless, several concerns have limited the utility of this technology. Most importantly, in cases where the desired transgene on the attP plasmid is a modified version of a parasite gene, the endogenous copy is not affected by transfection and is expected to remain functional, yielding a merodiploid whose phenotype may be more difficult to study. Expression levels for the transgene may also confound interpretation: genomic site effects due to expression from a heterologous cg6 locus or concatemerization of the attP plasmid prior to integration may lead to unanticipated transcript levels. Another concern is that the phenotype under study may be affected by disruption of the cg6 gene. Production of lines that carry attB at nonessential loci other than cg6 could address some of these concerns, but this has not been undertaken to our knowledge.

While Cre recombinase, a distinct phage recombinase from the tyrosine integrase family [33], has been used in various parasites [34-36], use of Bxb1 integrase has not been described for Apicomplexan parasites other than Plasmodium spp.

\section{Integrase strategy for native gene manipulation in Plasmodium falciparum}

To address these limitations and enable rapid and essentially unlimited manipulation of an endogenous parasite gene, we devised and used a modified version of this Bxb1 integrase strategy [17]. Our approach entails introducing attB into an intron of the gene of interest (Fig. $1 \mathrm{~b}$ ); we found that this relatively short element did not adversely affect mRNA splicing, transcription or translation of clag3, an intensively studied gene linked to malaria parasite nutrient uptake [37-39]. As changes to the intronic sequence are silent, the encoded protein should be faithfully translated without mutation or changes in associated phenotypes. Consistent with this prediction, our studies with the clag3 target revealed expression of a full-length unmodified protein, unchanged trafficking of the encoded protein to the host membrane, and preserved channel-mediated solute uptake and pharmacology [17]. As knockdown or modification of the clag3 gene product is associated with a significant fitness cost $[40,41]$, these findings suggest that most essential genes will tolerate addition of an intronic attB sequence.

We envision that this approach will be broadly applicable to study of $P$. falciparum genes. Approximately half of this parasite's genes have one or more introns with a small average size, $179 \mathrm{bp}$ [42], simplifying addition of attB and attP sequences. This cloning step can be performed either through DNA synthesis or through hybridization of complementary oligonucleotides in cases where the introns are small. Consistent with previous additions of short sequences to this parasite's introns [43], these small silent insertions are generally well-tolerated in $P$. falciparum. Although we have not examined the effect of position within the intron systematically, we suggest a central placement within the intron and preferably at least 60 nucleotides upstream of the 3' end of the intron as splicing branch points have been observed up to 57 nucleotides from the 3' intron-exon boundary [42]. For genes that lack introns, insertion of a heterologous intron carrying the $a t t B$ sequence into the open reading frame (ORF) offers excellent prospects for introduction of a silent $a t t B$ without requiring modifying the encoded protein's sequence; our clag3 attB intron has been validated and should work in most cases based on studies confirming faithful recognition and splicing of heterologous introns [43].

We originally introduced the attB-loaded intron into clag3 via single crossover recombination, but this may be simplified by CRISPR-Cas9 transfection with a donor plasmid that contains homology arms of 200-300 bp on either side of the intron; if CRISPR-Cas9 is used, it is critical to select the single guide RNA (sgRNA) and cleavage site carefully with proximity to the intron and on-target efficiency score the most critical factors in $P$. falciparum transfections [44]. After parasites grow out 
in this first transfection, it is important to use limiting dilution cloning to obtain a clonal population carrying the $a t t B$ sequence. Although some workers have argued that limiting dilution cloning is not required because subpopulations without this integration event will be removed by cloning after the second transfection, we caution against this because genome-level modifications at non-target sites may grow preferentially in the second transfection, aggravating attempts to identify the desired final clone.

After the silent attB site has been successfully introduced, the full range of target gene modifications can be generated with a second transfection with two plasmids (Fig. 2a, pLN-attP and pINT). The pLN-attP plasmid carries the attP element followed by the distal intronic sequence with a 3' splice site for faithful splicing after integration. Any sequence following this attP-intron will be inserted into the genome upon attB-attP recombination as facilitated by the Bxb1 integrase expressed from the pINT helper plasmid.

The use of a silent attB in a target gene's intron distinguishes our approach from previous use of this technology in malaria research and has two important consequences. It disrupts the target gene by inserting the entire pLN-attP plasmid in the gene after the modified intron. It also achieves a promoter-trap because the sequence inserted distal to the intron upon recombination will be spliced in frame behind the native promoter and gene sequence upstream of the intron. Thus, the outcome in most cases is an effective gene replacement.

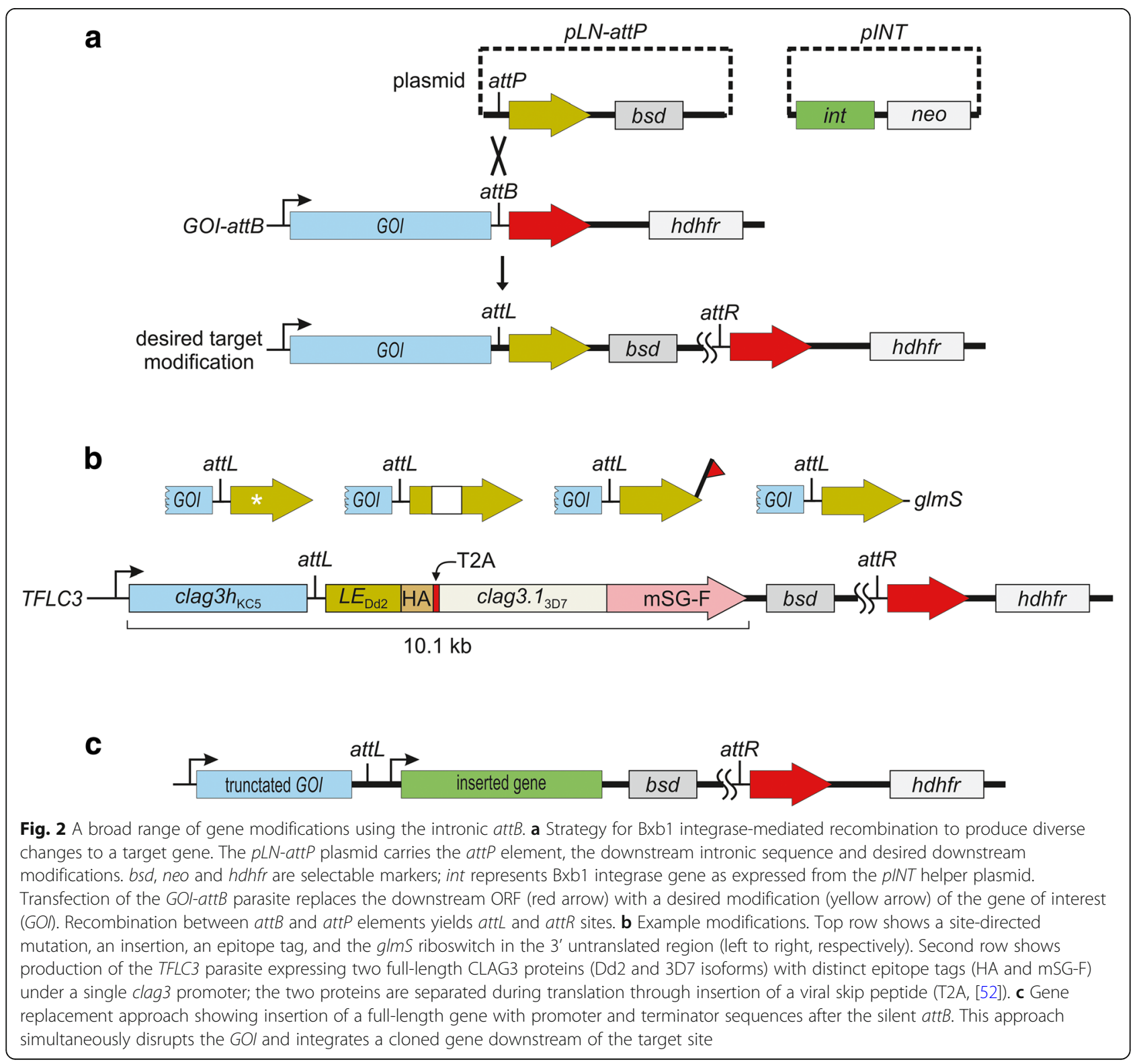


In one application, this distal sequence could be the remainder of the gene with desired modifications. These modifications may include site-directed mutations, internal insertions or deletions (indels), domain swaps, addition of C-terminal epitope tags on the encoded protein, modified codon usage, and/or altered transcriptional regulatory elements (e.g. removal of distal introns or addition of the glmS riboswitch into the 3' untranslated region). To demonstrate the range of possible modifications, we reported the production of a merodiploid parasite that expresses two CLAG3 proteins with distinct functional properties and separate epitope tags using this approach; although these clag3 genes are large (5.3 $\mathrm{kb}$ each) and we desired distinct epitope tags on two proteins expressed under a single promoter, our strategy and use of the clag3-attB line permitted facile introduction with a single plasmid (Fig. 2b, TFLC3; [17]).

In another application, the promoter trap enabled by the silent attB-intron is disregarded, and the pLN-attP plasmid may carry an entire gene cassette with required 5' and 3' untranslated regions (Fig. 2c). This may be particularly useful for examining whether other genes (e.g. paralogs) can adequately replace essential activities of the target gene carrying the attB-intron. For such applications, an important caveat is that the sequence upstream of the intron will typically still be transcribed and translated, yielding a truncated protein. For large genes such as clag3, the effect of the residual upstream protein may be significant, yielding either preserved activity or a dominant-negative effect due to protein-protein interactions with the truncated protein. This theoretical concern may be largely avoided by introducing the attB into an intron near the start of the target gene. If such an intron is not available, a heterologous attB-containing intron may be inserted at any desired position as described above.

\section{Comparison to other methods available for targeted gene modifications}

While there are several methods for expression of transgenes in P. falciparum [45], there are only a few options for directed modification of a target gene of interest. Table 1 summarizes these options along with the required components, advantages and limitations of each method. Although CRISPR-Cas9 is the most straightforward to implement and allows rapid gene editing suitable for most applications [11, $12,44]$, use of Bxb1 integrase with an engineered intronic $a t t B$ site may be preferred for large insertions and has several other advantages.

Our use of an intronic attB site resembles the recent description of loxP insertion into introns for conditional editing of a target gene [43], but there are important differences that confer greater versatility to attB-attP technology. Both use a target gene intron to introduce short DNA elements that enable enzyme-mediated DNA recombination. The DiCre-loxP system sites uses two halves of Cre recombinase linked to distinct rapamycin binding proteins; addition of rapamycin stimulates dimerization of the two halves, allowing conditional Cre activity and recombination at two properly oriented loxP sites. This system permits conditional knockout or modification such as addition of an epitope tag or site-directed mutation [43]. The main strength of this system relative to our att$B$-intron system is that it allows conditional modifications dependent on small molecule addition. At the same time, a key limitation is that facilitated recombination between the two $\operatorname{lox} P$ sites is reversible, in contrast to irreversible recombination between $a t t B$ and $a t t P$ sites [26]. This reversibility prevents use of a single initial transfectant with a loxP-intron for multiple distinct modifications: a plasmid carrying a loxP with desired distal modifications, analogous to our pLN-attP plasmid (Fig. 2a), would likely undergo cycles of integration and excision due to the reversibility of loxP recombination. This results in an inefficient integration reaction; although various strategies have been devised to overcome this inefficiency [46], these have not been implemented in malaria research to our knowledge. Thus, multiple distinct modifications cannot be performed with a single engineered loxP parasite: each desired modification requires a de novo transfection to introduce the loxP-intron cassette into the parasite genome.

As increasingly acknowledged by workers, CRISPRCas9 technology will be the method of choice for most gene modifications in P. falciparum. This system largely bypasses the need for introduction of DNA motifs such as $a t t B$ or attP; it requires expression of Cas9, an RNA-guided endonuclease, and a small single-stranded RNA molecule known as the single guide RNA or sgRNA to achieve site-specific modification of the genome [47]. The sgRNA binds Cas9 and identifies the cleavage site through Watson-Crick base pairing over a 20 bp recognition sequence; gene editing results from blunt-end cleavage within the recognition sequence. Although a protospacer adjacent domain (PAM) must be present immediately after the chosen recognition sequence, there are nearly 663,000 canonical PAM sites in the P. falciparum genome [44]; thus, almost any genomic site can be targeted. Nevertheless, we considered two important cases where CRISPR-Cas9 may not be the ideal method for target gene modifications. One problematic situation arises when a gene of interest is a member of a multigene family. If the site to be modified in the target gene is conserved amongst paralogs that should not be edited, it may be difficult to find an sgRNA that does not cleave one or more of these paralogs; such "off-target" cleavage sites may be predicted and avoided with a newly devised paralog specificity score [44]. If multiple modifications of a target gene are envisioned, use of the CRISPR system may still require 


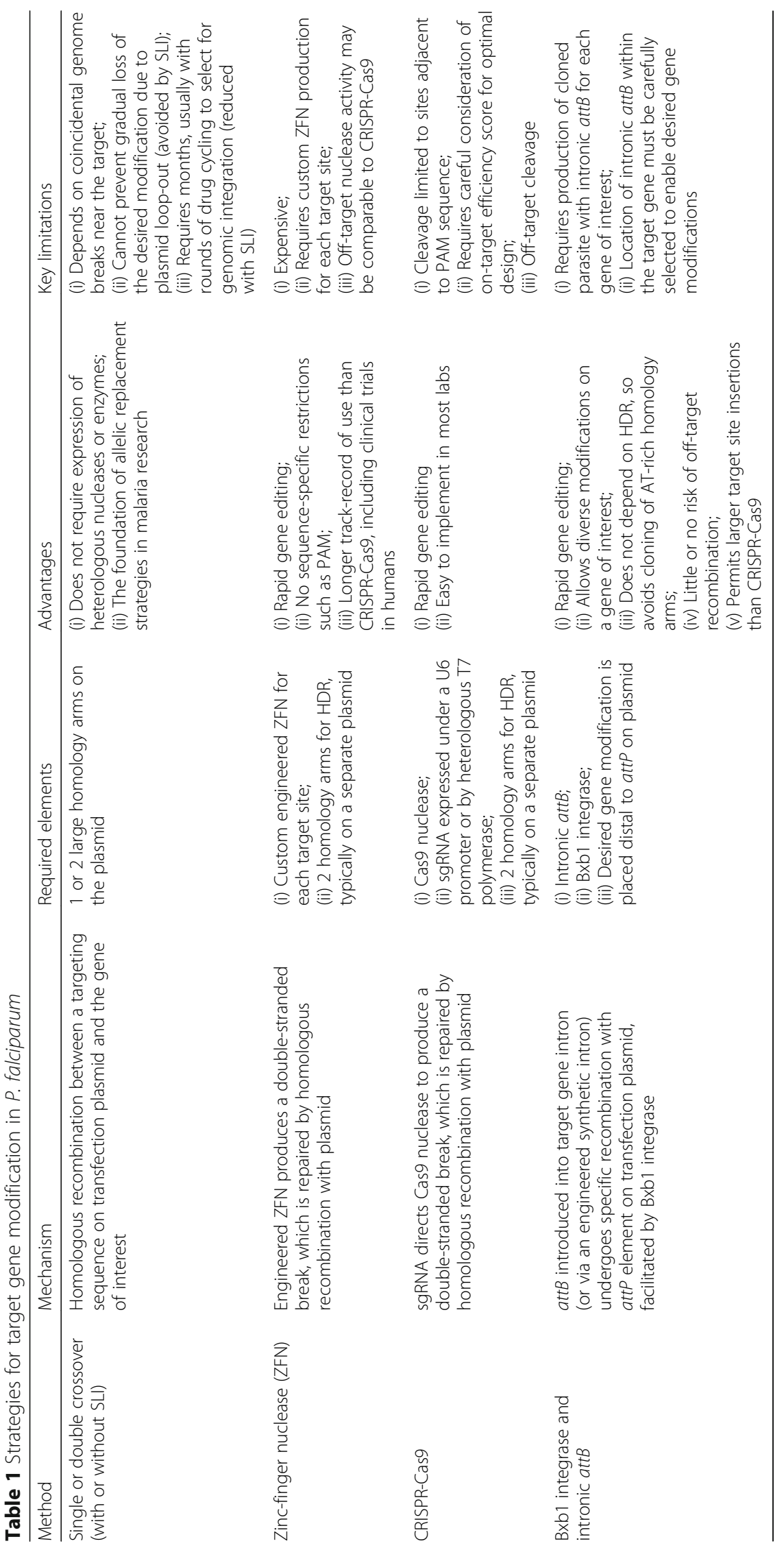


greater effort and cost because confident production of each modification will require screening of limiting dilution clones to identify parasites with the desired target gene modification and no unwanted edits to paralogs. The attB-intron strategy reduces labor and expense because the identification of a clone without unwanted $a t t B$ introduction into paralogs need only be done once: subsequent transfections with attP-containing plasmids will then be highly specific for the desired target gene, confidently avoiding paralogs.

Even for genes not within multigene families, off-target effects remain a concern with CRISPR-Cas9 editing because the Cas9 nuclease can recognize and cleave at sites carrying one or more mismatches relative to the selected $20 \mathrm{bp}$ recognition sequence. This limitation has led to the development of predictive off-target scoring algorithms and Cas9 mutants with ongoing improvements in specificity $[16,48]$. The targeted genome break produced by Cas 9 nuclease may also lead to chromosome repair by production of large deletions or more complex rearrangements [49], although these have not been reported to date in malaria research. Promiscuous recombination and target site rearrangements have not been reported with the Bxb1 integrase, presumably because of the highly coordinated attB-attP recombination process [50].

Another important case where the attB-intron strategy will be preferred over technologies such as CRISPR-Cas9 and selection-linked integration (SLI, [51]) is exemplified by the production of a parasite carrying two clag3 genes under the native promoter (TLFC3 parasite in Fig. 2b). SLI selects specifically for homologous recombination at the target site by using a promoter trap to drive expression of the selectable marker gene. Because both CRISPR-Cas9 and SLI depend on homologous recombination, both would require fully re-codonized versions of the two clag3 genes to produce TFLC3 to avoid internal recombination events that bypass introduction of desired tags on the two clag3 alleles ("HA" and "mSG-F" in Fig. 2b TFLC3 ribbon). This re-codonization would require custom synthesis of a $6.3 \mathrm{~kb}$ DNA, which is both prohibitively expensive and time-consuming for most research labs.

\section{Conclusions}

DNA transfection technologies represent an important tool for basic and translational malaria research. We remain limited by a low transfection efficiency, relatively slow parasite replication, a paucity of selection markers, and other difficulties specific to this important pathogen. Ongoing improvements in the methods available for generating parasite lines with desired genome modifications make this is an exciting time for malaria research. While CRISPR-Cas9 is currently the method-of-choice for most gene editing experiments, other strategies such as our attB-intron method should be considered, especially for labs that have committed to specific genes and require a broad collection of gene modifications for biochemical and/ or structural studies.

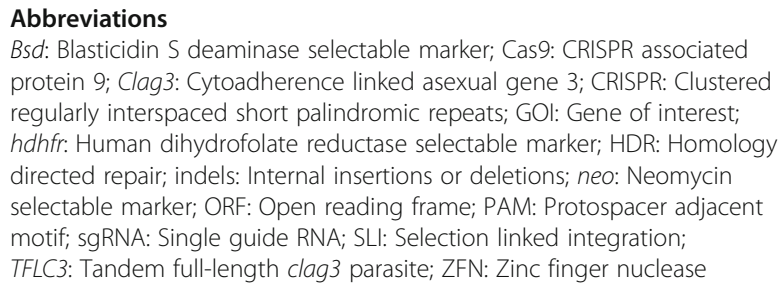

Bsd: Blasticidin S deaminase selectable marker; Cas9: CRISPR associated protein 9; Clag3: Cytoadherence linked asexual gene 3; CRISPR: Clustered regularly interspaced short palindromic repeats; GOI: Gene of interest; hdhfr: Human dihydrofolate reductase selectable marker; HDR: Homology directed repair; indels: Internal insertions or deletions; neo: Neomycin selectable marker; ORF: Open reading frame; PAM: Protospacer adjacent motif; sgRNA: Single guide RNA; SLI: Selection linked integration; TFLC3: Tandem full-length clag3 parasite; ZFN: Zinc finger nuclease

\section{Funding}

This work was supported by the Intramural Research Program of National Institutes of Health $(\mathrm{NIH})$, National Institute of Allergy and Infectious Diseases (NIAID), USA.

\section{Availability of data and materials}

The materials and other data generated from this study are available from the corresponding author upon reasonable request.

\section{Authors' contributions}

PBN drafted the first version of the manuscript. Both authors edited, read and approved the final manuscript.

Ethics approval and consent to participate

Not applicable.

\section{Consent for publication}

Not applicable.

\section{Competing interests}

The authors declare that they have no competing interests.

\section{Publisher's Note}

Springer Nature remains neutral with regard to jurisdictional claims in published maps and institutional affiliations.

\section{Author details}

${ }^{1}$ The Laboratory of Malaria and Vector Research, National Institute of Allergy and Infectious Diseases, National Institutes of Health, Rockville, MD 20852, USA. ${ }^{2}$ Present Address: Department of Epidemiology and Public Health, Central University of Tamil Nadu, Thiruvarur, India.

Received: 5 June 2018 Accepted: 1 October 2018

Published online: 17 October 2018

\section{References}

1. Waterkeyn JG, Crabb BS, Cowman AF. Transfection of the human malaria parasite Plasmodium falciparum. Int J Parasitol. 1999;29:945-55.

2. Gardiner DL, Skinner-Adams TS, Spielmann T, Trenholme KR. Malaria transfection and transfection vectors. Trends Parasitol. 2003;19:381-3.

3. Balu B, Adams JH. Advancements in transfection technologies for Plasmodium. Int J Parasitol. 2007;37:1-10.

4. Mamoun CB, Gluzman IY, Goyard S, Beverley SM, Goldberg DE. A set of independent selectable markers for transfection of the human malaria parasite Plasmodium falciparum. Proc Natl Acad Sci USA. 1999;96:8716-20.

5. Crabb BS, Rug M, Gilberger TW, Thompson JK, Triglia T, Maier AG, et al. Transfection of the human malaria parasite Plasmodium falciparum. Methods Mol Biol. 2004;270:263-76.

6. Kadekoppala M, Cheresh P, Catron D, Ji D, Deitsch K, Wellems TE, et al. Rapid recombination among transfected plasmids, chimeric episome formation and trans gene expression in Plasmodium falciparum. Mol Biochem Parasitol. 2001;112:211-8. 
7. O'Donnell RA, Preiser PR, Williamson DH, Moore PW, Cowman AF, Crabb BS. An alteration in concatameric structure is associated with efficient segregation of plasmids in transfected Plasmodium falciparum parasites. Nucleic Acids Res. 2001;29:716-24.

8. Balu B, Adams JH. Functional genomics of Plasmodium falciparum through transposon-mediated mutagenesis. Cell Microbiol. 2006:8:1529-36.

9. Nkrumah LJ, Muhle RA, Moura PA, Ghosh P, Hatfull GF, Jacobs WR Jr, Fidock DA. Efficient site-specific integration in Plasmodium falciparum chromosomes mediated by mycobacteriophage Bxb1 integrase. Nat Methods. 2006;3:615-21.

10. Straimer J, Lee MC, Lee AH, Zeitler B, Williams AE, Pearl JR, et al. Site-specific genome editing in Plasmodium falciparum using engineered zinc-finger nucleases. Nat Methods. 2012;9:993-8.

11. Ghorbal M, Gorman M, Macpherson CR, Martins RM, Scherf A, Lopez-Rubio J. Genome editing in the human malaria parasite Plasmodium falciparum using the CRISPR-Cas9 system. Nat Biotechnol. 2014;32:819-21.

12. Wagner JC, Platt RJ, Goldfless SJ, Zhang F, Niles JC. Efficient CRISPR-Cas9-mediated genome editing in Plasmodium falciparum. Nat Methods. 2014;11:915-8

13. Veiga MI, Dhingra SK, Henrich PP, Straimer J, Gnadig N, Uhlemann AC, et al. Globally prevalent PfMDR1 mutations modulate Plasmodium falciparum susceptibility to artemisinin-based combination therapies. Nat Commun. 2016;7:11553.

14. Ito D, Schureck MA, Desai SA. An essential dual-function complex mediates erythrocyte invasion and channel-mediated nutrient uptake in malaria parasites. Elife. 2017;6:e23485.

15. Bansal A, Molina-Cruz A, Brzostowski J, Mu J, Miller LH. Plasmodium falciparum calcium-dependent protein kinase 2 is critical for male gametocyte exflagellation but not essential for asexual proliferation. MBio. 2017:8:e01656-17.

16. Doench JG, Fusi N, Sullender M, Hegde M, Vaimberg EW, Donovan KF, et al. Optimized sgRNA design to maximize activity and minimize off-target effects of CRISPR-Cas9. Nat Biotechnol. 2016;34:184-91.

17. Gupta A, Balabaskaran-Nina P, Nguitragool W, Saggu GS, Schureck MA Desai SA. CLAG3 self-associates in malaria parasites and quantitatively determines nutrient uptake channels at the host membrane. mBio. 2018;9: e02293-17.

18. Armstrong CM, Goldberg DE. An FKBP destabilization domain modulates protein levels in Plasmodium falciparum. Nat Methods. 2007:4:1007-9.

19. Muralidharan V, Oksman A, Iwamoto M, Wandless TJ, Goldberg DE. Asparagine repeat function in a Plasmodium falciparum protein assessed via a regulatable fluorescent affinity tag. Proc Natl Acad Sci USA. 2011; 108:4411-6

20. Ganesan SM, Falla A, Goldfless SJ, Nasamu AS, Niles JC. Synthetic RNAprotein modules integrated with native translation mechanisms to control gene expression in malaria parasites. Nat Commun. 2016;7:10727.

21. Prommana $\mathrm{P}$, Uthaipibull C, Wongsombat C, Kamchonwongpaisan S, Yuthavong Y, Knuepfer E, et al. Inducible knockdown of Plasmodium gene expression using the glmS ribozyme. PLoS One. 2013;8:e73783.

22. Kim Al, Ghosh P, Aaron MA, Bibb LA, Jain S, Hatfull GF. Mycobacteriophage Bxb1 integrates into the Mycobacterium smegmatis groEL1 gene. Mol Microbiol. 2003;50:463-73.

23. Groth AC, Calos MP. Phage integrases: biology and applications. J Mol Biol 2004;335:667-78

24. Ghosh P, Bibb LA, Hatfull GF. Two-step site selection for serine-integrasemediated excision: DNA-directed integrase conformation and central dinucleotide proofreading. Proc Natl Acad Sci USA. 2008;105:3238-43.

25. Rutherford K, Van Duyne GD. The ins and outs of serine integrase sitespecific recombination. Curr Opin Struct Biol. 2014;24:125-31.

26. Ghosh P, Wasil LR, Hatfull GF. Control of phage Bxb1 excision by a novel recombination directionality factor. PLoS Biol. 2006:4:e186.

27. Rutherford K, Yuan P, Perry K, Sharp R, Van Duyne GD. Attachment site recognition and regulation of directionality by the serine integrases. Nucleic Acids Res. 2013:41:8341-56.

28. Keenholtz RA, Grindley ND, Hatfull GF, Marko JF. Crossover-site sequence and DNA torsional stress control strand interchanges by the Bxb1 site-specific serine recombinase. Nucleic Acids Res. 2016;44:8921-32.

29. Olorunniji FJ, McPherson AL, Rosser SJ, Smith MCM, Colloms SD, Stark WM. Control of serine integrase recombination directionality by fusion with the directionality factor. Nucleic Acids Res. 2017;45:8635-45.
30. Rowley PA, Smith MC, Younger E, Smith MC. A motif in the C-terminal domain of phiC31 integrase controls the directionality of recombination. Nucleic Acids Res. 2008;36:3879-91.

31. Farruggio AP, Calos MP. Serine integrase chimeras with activity in E. coli and HeLa cells. Biol Open. 2014;3:895-903.

32. Ke H, Morrisey JM, Ganesan SM, Painter HJ, Mather MW, Vaidya AB. Variation among Plasmodium falciparum strains in their reliance on mitochondrial electron transport chain function. Eukaryot Cell. 2011;10:1053-61.

33. Fogg PC, Colloms S, Rosser S, Stark M, Smith MC. New applications for phage integrases. J Mol Biol. 2014;426:2703-16.

34. Brecht S, Erdhart $H$, Soete M, Soldati D. Genome engineering of Toxoplasma gondii using the site-specific recombinase Cre. Gene. 1999;234:239-47.

35. Santos RERS, Silva GLA, Santos EV, Duncan SM, Mottram JC, Damasceno JD, et al. A DiCre recombinase-based system for inducible expression in Leishmania major. Mol Biochem Parasitol. 2017;216:45-8.

36. Wampfler PB, Faso C, Hehl AB. The Cre/loxP system in Giardia lamblia: genetic manipulations in a binucleate tetraploid protozoan. Int J Parasitol. 2014:44:497-506.

37. Nguitragool W, Bokhari AA, Pillai AD, Rayavara $K$, Sharma $P$, Turpin $B$, et al. Malaria parasite clag3 genes determine channel-mediated nutrient uptake by infected red blood cells. Cell. 2011;145:665-77.

38. Rovira-Graells N, Crowley VM, Bancells C, Mira-Martinez S, Ribas de PL, Cortes A. Deciphering the principles that govern mutually exclusive expression of Plasmodium falciparum clag3 genes. Nucleic Acids Res. 2015; 43:8243-57.

39. Nguitragool W, Rayavara K, Desai SA. Proteolysis at a specific extracellular residue implicates integral membrane CLAG3 in malaria parasite nutrient channels. PLoS One. 2014;9:e93759.

40. Sharma P, Wollenberg K, Sellers M, Zainabadi K, Galinsky K, Moss E, et al. An epigenetic antimalarial resistance mechanism involving parasite genes linked to nutrient uptake. J Biol Chem. 2013:288:19429-40.

41. Sharma P, Rayavara K, Ito D, Basore K, Desai SA. A CLAG3 mutation in an amphipathic transmembrane domain alters malaria parasite nutrient channels and confers leupeptin resistance. Infect Immun. 2015;83:2566-74.

42. Zhang X, Tolzmann CA, Melcher M, Haas BJ, Gardner MJ, Smith JD, et al. Branch point identification and sequence requirements for intron splicing in Plasmodium falciparum. Eukaryot Cell. 2011;10:1422-8.

43. Jones ML, Das S, Belda H, Collins CR, Blackman MJ, Treeck M. A versatile strategy for rapid conditional genome engineering using loxP sites in a small synthetic intron in Plasmodium falciparum. Sci Rep. 2016;6:21800.

44. Ribeiro JM, Garriga M, Potchen N, Crater AK, Gupta A, Ito D, et al. Guide RNA selection for CRISPR-Cas9 transfections in Plasmodium falciparum. Int J Parasitol. 2018. https://doi.org/10.1016/j.ijpara.2018.03.009.

45. de Koning-Ward TF, Gilson PR, Crabb BS. Advances in molecular genetic systems in malaria. Nat Rev Microbiol. 2015;13:373-87.

46. Araki K, Araki M, Yamamura K. Site-directed integration of the cre gene mediated by Cre recombinase using a combination of mutant lox sites. Nucleic Acids Res. 2002;30:e103.

47. Wright AV, Nunez JK, Doudna JA. Biology and applications of CRISPR systems: harnessing nature's toolbox for genome engineering. Cell. 2016; 164:29-44.

48. Chen JS, Dagdas YS, Kleinstiver BP, Welch MM, Sousa AA, Harrington LB, et al. Enhanced proofreading governs CRISPR-Cas9 targeting accuracy. Nature. 2017:550:407-10.

49. Kosicki M, Tomberg K, Bradley A. Repair of double-strand breaks induced by CRISPR-Cas9 leads to large deletions and complex rearrangements. Nat Biotechnol. 2018:36:765-71.

50. Singh S, Ghosh P, Hatfull GF. Attachment site selection and identity in Bxb1 serine integrase-mediated site-specific recombination. PLoS Genet. 2013;9: e1003490.

51. Birnbaum J, Flemming S, Reichard N, Soares AB, Mesen-Ramirez $P$, Jonscher E, et al. A genetic system to study Plasmodium falciparum protein function. Nat Methods. 2017;14:450-6.

52. Kim JH, Lee SR, Li LH, Park HJ, Park JH, Lee KY, et al. High cleavage efficiency of a 2A peptide derived from porcine teschovirus-1 in human cell lines, zebrafish and mice. PLoS One. 2011;6:e18556. 